Chirurg 2018 89:1-3

https://doi.org/10.1007/s00104-017-0562-5

(C) Springer Medizin Verlag GmbH, ein Teil von Springer Nature 2017

CrossMark

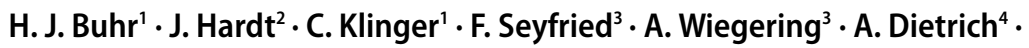

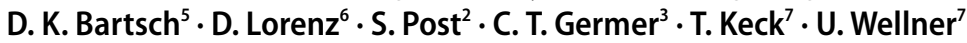
' Deutsche Gesellschaft für Allgemein- und Viszeralchirurgie, Berlin, Deutschland; ${ }^{2}$ Chirurgische Klinik, Universitätsmedizin Mannheim, Mannheim, Deutschland; ${ }^{3}$ Klinik für Allgemein-, Viszeral-, Gefäß- und Kinderchirurgie, Universitätsklinikum Würzburg, Würzburg, Deutschland; ${ }^{4}$ Klinik und Poliklinik für Viszeral-, Transplantations-, Thorax- und Gefäßchirurgie Bereich Bariatrische Chirurgie,

Universitätsklinikum Leipzig, Leipzig, Deutschland; ${ }^{5}$ Klinik für Visceral-, Thorax- und Gefäßchirurgie, Universitätsklinikum Marburg, Marburg, Deutschland; ${ }^{6}$ Klinik für Allgemein- und Viszeralchirurgie bzw. der Medizinischen Klinik II Gastroenterologie, Hepatologie, Endokrinologie, Infektiologie, Sana Klinikum Offenbach, Offenbach, Deutschland; ' ${ }^{7}$ Klinik für Allgemeine Chirurgie, Universitätsklinikum S.-H. Campus Lübeck, Lübeck, Deutschland

\section{Qualitätsindikatoren mit Referenz- und Grenzwerten in der Allgemein- und Viszeralchirurgie}

\section{Für die Adipositas- und metabolische, Pankreas-, Kolonkarzinom- und Rektumkarzinomchirurgie}

Die Deutsche Gesellschaft für Allgemeinund Viszeralchirurgie (DGAV) mit über 5100 Mitgliedern sieht eine wichtige Aufgabe darin, Hilfestellung für eine permanente Qualitätsverbesserung der chirurgischen Ergebnisse zu geben und damit dem Patientenwohl zu dienen und zur Verbesserung der Lebensqualität der Patienten beizutragen. Dieser Auftrag ist auch in der Satzung der DGAV $₫ 2$ (5) festgehalten: „Mitwirkung an Maßnahmen der Qualitätssicherung und Qualitätsverbesserung“.

Voraussetzung für eine Analyse der chirurgischen Qualität, ihrer Sicherung und einer ggf. vorzunehmenden Qualitätsverbesserung ist die Formulierung chirurgischer Qualität! Was ist chirurgische Qualität? Wie soll sie definiert und erfasst werden, um auch Vergleiche zwischen verschiedenen Krankenhäusern (Benchmarks) anstellen zu können? Welche Faktoren fließen in das chirurgische Ergebnis ein und welche Einflussgrößen müssen berücksichtigt werden? Diese wenigen Punkte zeigen bereits, wie schwierig es ist, chirurgische
Qualität objektiv zu beurteilen. Auch wenn chirurgische Qualität voreilig mit der chirurgischen Handlung im Operationssaal gleichgesetzt wird, müssen hier doch auch Strukturen und Prozessabläufe in den betreffenden Krankenhäusern berücksichtigt werden.

In den letzten Jahren hat sowohl die Gesundheitspolitik als auch die informierte Öffentlichkeit den Fokus auf die Qualitätssicherung in der Medizin im Allgemeinen und in der Chirurgie im Speziellen gerichtet. Für Chirurgen ist und war es schon immer ein „imperatives Muss“, intensiv die chirurgische Qualitätssicherung zu betreiben.

) Anforderungen an QI sind: Relevanz, Wissenschaftlichkeit und Praktikabilität

Nach Angaben des Ärztlichen Zentrums für Qualität in der Medizin (ÄZQ; [1]) sind Qualitätsindikatoren (QI) Maße, welche nach ihrer Ausprägung eine Unterscheidung zwischen guter und 
schlechter Qualität ermöglichen. Qualitätsindikatoren messen hierbei Qualität nicht direkt, sondern sind Surrogatparameter für Qualität [2]. Als solche sollten sie gemäß den sog. QUALIFYKriterien Anforderungen der folgenden Kategorien genügen: Relevanz, Wissenschaftlichkeit und Praktikabilität. In die Kategorie Relevanz fallen Aspekte wie Bedeutung für das Versorgungssystem und Nutzen, die Wissenschaftlichkeit ergibt sich u. a. aus der Klarheit der Definition und vorhandenen Evidenz und die Praktikabilität folgt aus der Verständlichkeit für Fachpersonal, Patienten und Öffentlichkeit, der Datenverfügbarkeit und -überprüfbarkeit sowie dem Erhebungsaufwand. Da einzelne QI als Surrogatparameter die Qualität naturgemäß nur teilweise abbilden, wird die Verwendung mehrerer QI im Sinne von QI-Profilen empfohlen (ÄZQ [1]).

Chirurgische Qualität umfasst somit

- Strukturqualität

- Prozessqualität

- Ergebnisqualität

\section{》) Literatur und die StuDoQ- Register der DGAV wurden berücksichtigt}

Chirurgische Qualität lässt sich anhand definierter QI festlegen, welche die drei genannten Bereiche abbilden. Nach der Auswahl von QI für einzelne Krankheitsbilder und ihrer operativen Behandlung aus einer Vielzahl möglicher Indikatoren, besteht der nächste komplexe Schritt in der Festlegung der Referenzbzw. der Grenzwerte. Diese Festlegung auf Referenz- und Grenzwerte kann aber nur dann zur Qualitätssicherung beitragen, wenn sie von Chirurgen auch anerkannt und angewendet wird. Voraussetzung hierfür sind nicht willkürlich festgelegte Werte, die aus Abrechnungsdaten gewonnen werden, sondern dass die Risikofaktoren des Patienten berücksichtigt werden, d.h. wenn die präoperative Morbidität, die ja einen hohen Einfluss auf das postoperative chirurgische Ergebnis hat, in der Berechnung der Referenz- und Grenzwerte einbezogen wird. Ohne eine notwendige
Risikoadjustierung sollten keine Werte für eine Beurteilung der Ergebnisqualität herangezogen werden. Die DGAV führt in ihren StuDoQ(Studien-, Dokumentations- und Qualitätszentrum)Registern eine Risikoadjustierung durch. Beim Kolon- und Rektumkarzinom werden so z. B. 40 Risikofaktoren bei der Berechnung der 20 QI berücksichtigt.

Vor dem Hintergrund dieser dargestellten Herausforderungen hat die DGAV mehrere Arbeitsgruppen eingerichtet, die sich mit der Evaluierung und Formulierung von QI und der einzelnen Referenz- und Grenzwerte auseinandersetzen sollten. Nach einem Vorstandsbeschluss wurden Arbeitsgruppen gebildet für Erkrankungen, für welche die DGAV StuDoQ-Register führt:

- Adipositas

- Kolonkarzinom

- Rektumkarzinom

- Pankreaskarzinom

Die Teilnehmer dieser Arbeitsgruppen setzten sich aus DGAV-Vorstandsmitgliedern, Vorstandsmitgliedern der betreffenden Arbeitsgemeinschaften und aus chirurgischen Abteilungen, die zahlreiche Fälle in die einzelnen StuDoQRegister eintragen, zusammen. Die Arbeitsgruppen haben sich mehrmals zu ganztägigen Besprechungen und Diskussionen versammelt.

Die Literaturrecherche erfolgte durch Vertreter der beteiligten Kliniken

- Adipositas: PD Dr. F. Seyfried, Würzburg

- Kolonkarzinom: Dr. J. Hardt, Mannheim

- Rektumkarzinom: PD Dr. A. Wiegering, Würzburg

- Pankreaskarzinom: PD Dr. U. Wellner, Lübeck

Nach den Vorgaben des „Manual Systematische Literaturrecherche für die Erstellung von Leitlinien“, das vom ÄZQ [3], von der Arbeitsgemeinschaft der Wissenschaftlichen Medizinischen Fachgesellschaften (AWMF) und vom Deutschen Cochrane-Zentrum (DCZ) zusammengestellt wurde sowie den Vorgaben des Gemeinsamen Bundesauschusses (GBA) erfolgte eine ausführliche Literaturrecherche in der PubMed Datenbank.
Die Arbeitsgruppen haben für die einzelnen Erkrankungen bzw. Operationsverfahren Suchbegriffe, eventuell zur Verfügung stehende Qualitätsindikatoren definiert. Für die Erstellung von Referenzwerten wurden nur populationsbasierte und prospektive Studien der letzten 10 Jahre mit englischem Abstrakt zur weiteren Begutachtung herangezogen.

Gleichzeitig erfolgte die Analyse in den entsprechenden StuDoQ-Registern. Die Daten für die einzelnen Suchbegriffe (Indikatoren) wurden ausgewertet und mit den Daten der Literatur verglichen. Nach Erarbeitung der Literatur und der StuDoQ-Register über die betreffenden Suchbegriffe (Indikatoren) wurden in ausführlichen Diskussionen in den betreffenden Arbeitsgruppen die jetzt im Weiteren verwandten und praktikablen QI bestimmt.

\section{》) Die Festlegung der Referenz- und Grenzwerte war ein intensiver Prozess}

Gleichzeitig wurden die Referenz- und Grenzwerte für die einzelnen QI festgelegt. Die Benennung der Referenz- und Grenzwerte war ein besonders aufwendiger Prozess. Wenn eine Einigung auf einen bestimmten Wert nicht zustande kam, erfolgte die Abstimmung in der Arbeitsgruppe und anschließend im Plenum (aller Teilnehmer).

Bei der Bestimmung der Referenzund Grenzwerte war die Literatur unterschiedlich zu bewerten. Häufig wurden wichtige Fragestellungen in den Arbeiten nicht erwähnt, oder es sind wie z. B. bei der Liegedauer Vergleiche mit anderen Ländern nicht möglich. Daher wurde bei der Festlegung der Werte die Datenanalyse aus den StuDoQ-Registern herangezogen, um auch die spezifischen deutschen Verhältnisse zu berücksichtigen.

Nach der Festlegung der QI und ihrer Referenz- und Grenzwerte in den einzelnen Arbeitsgruppen erfolgte eine weitere Diskussion in Bezug auf Logik, Anwendung bzw. Praktikabilität der einzelnen QI in den Plenarsitzungen (insgesamt vier Sitzungen), an denen die Teilnehmer aller Arbeitsgruppen beteiligt waren. 
Ziel ist es, nach einigen Jahren, die ausgesuchten Qualitätsindikatoren und ihre Referenz- und Grenzwerte erneut auf den Prüfstand zu stellen und den Prozess der QI-Formulierung in der Allgemein- und Viszeralchirurgie erneut durchlaufen $\mathrm{zu}$ lassen.
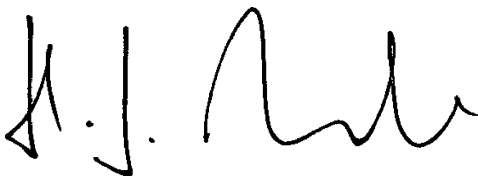

Prof. Dr. Heinz-J. Buhr

\section{Korrespondenzadresse}

\section{Prof. Dr. H. J. Buhr}

Deutsche Gesellschaft für Allgemein- und Viszeralchirurgie

Haus der Bundespressekonferenz,

Schiffbauerdamm 40, 10117 Berlin,

Deutschland

hbuhr@dgav.de

Interessenkonflikt. H. J. Buhr, J. Hardt, C. Klinger, F. Seyfried, A. Wiegering, A. Dietrich, D. K. Bartsch, D. Lorenz, S. Post, C. T. Germer, T. Keck und U. Wellner geben an, dass kein Interessenkonflikt besteht.

\section{Literatur}

1. Ärztliches Zentrum für Qualität in der Medizin (ÄZQ) (2009) Manual Qualitätsindikatoren. Manual für Autoren. ÄZQ Schriftenreihe, Bd. 36. ÄZQ, Berlin (http://www.aezq.de/edocs/pdf/schriftenreihe/ schriftenreihe.36.pdf)

2. Wiegering A, Wellner U, Seyfried F, Hardt J, Klinger C, Buhr H, Post S (2017) MTL30 als Surrogatparameter der Behandlungsqualität chirurgischer Erkrankungen. Etablierung anhand der StuDoQ-Register der Deutschen Gesellschaft für Allgemein- und Viszeralchirurgie. Chirurg. https://doi.org/10.1007/s00104-017-0479-z

3. Ärztliches Zentrum für Qualität in der Medizin (ÄZQ), Arbeitsgemeinschaft der Wissenschaftlichen Medizinischen Fachgesellschaften (AWMF) (2010) Deutsches Instrument zur methodischen Leitlinien-Bewertung (DELBI). http://www.delbi. de

\section{Germer, Christoph-Thomas, Keck, Tobias, Grundmann, Reinhart T. (Hrsg.) Evidenzbasierte Viszeralchirurgie benigner Erkrankungen}

Leitlinien und Studienlage

Berlin Heidelberg: Springer-Verlag Berlin Heidelberg 2017, 1. Auflage, 250 S., 3 Abb., (ISBN: 978-3-662-53552-3), Softcover+eBook 50,99 EUR, eBook

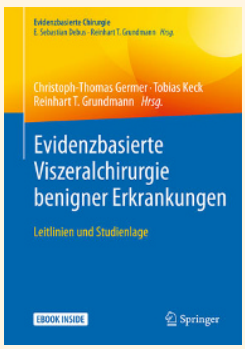

Die aktuelle leitliniengerechte, evidenzbasierte Behandlung häufiger Erkrankungen ist in der täglichen Praxis nicht immer bekannt. Für einzelne Entscheidungen bedarf es einer Interpretation, weil Leitlinien für die individuelle Situation des Patienten anwendbar sein sollten.

Diese Entscheidungshilfe und die Darstellung der "guten chirurgischen Praxis" für die Viszeralchirurgie ist das Anliegen dieses Buches.

Ein Abgleich mit Leitlinien anderer Länder (z.B. Japan, Niederlande, USA), anderer Fachgesellschaften, Metanalysen und Konsensusempfehlungen geben dem Inhalt eine hohe Evidenz.

Diese evidenzbasierten Therapieempfehlungen beziehen sich auf 17 viszeralchirurgische Indikationen bei benignen Erkrankungen. Häufige Eingriffe, wie Schilddrüsen- und Hernienoperationen, die Appendektomie, bariatrische Eingriffe, die Behandlung der Divertikulitis, das perforierte Magenulkus, die Hiatushernie, proktologische Operationen und die Problematik des akuten Abdomens werden, ebenso wie einige seltenere Eingrif$\mathrm{fe}$, hervorragend beschrieben.

Benigne Erkrankungen der Leber, der Milz, des Zwerchfells sowie die Therapie des lleus sollten in einer weiteren Auflage ergänzt werden.

Die Darstellung ist didaktisch nach den praktischen Fragestellungen ausgerichtet. Ein Fazit für die Praxis fasst am Ende jedes Kapitels den Erkenntnisstand und die Empfehlungen zusammen.

Ein kurzer Bezug zur Anamnese und zur Wertigkeit der klinischen Untersuchung als Voraussetzung für die ausführlich darge- stellte labor- und bildgebende Diagnostik in einigen Kapiteln ist gerade für jüngere

Mediziner hilfreich.

Das Buch ist eine ausgezeichnete Zusammenfassung von Diagnostik- und Therapieempfehlungen auf der Basis aktueller Leitlinien, Studien und Analysen und somit für die tägliche Praxis wertvoll.

H. Lippert (Magdeburg) 